\title{
Medidas zoométricas del ganado bovino criollo de Panamá ${ }^{1}$
}

\section{Cecilia de Escobar ${ }^{1, *}$, Axel Villalobos ${ }^{2}$ y Julivet Núñez ${ }^{1}$}

${ }^{1}$ Laboratorio de Análisis y Diagnóstico Molecular Agropecuario (LADMA), Instituto de Investigación Agropecuaria de Panamá (IDIAP), Panamá, República de Panamá

${ }^{2}$ Centro de Investigación Agropecuario Central (CIAC), Instituto de Investigación Agropecuaria de Panamá (IDIAP), Panamá, República de Panamá

*Autor para correspondencia. Email: ceciliadeescobar@hotmail.com

Recibido: 2 de diciembre de 2014

Aceptado: 9 de diciembre de 2014

\begin{abstract}
The aim of the study was to describe selected morphologic features in Creole cattle in Panama. During 2011, we evaluated 63 adult specimens, indigenous from three different locations: Gualaca (Chiriqui), El Valle de Anton (Cocle) and the Ngäbe-Buglé Comarca (Chiriquî). Nine zoometric variables were measured: face length (LC), face width (AC), thoracic perimeter (TP), total length (TL), height at withers (Acr), rump height (AG), body length $(\mathrm{LgCu})$, hip length $(\mathrm{LgCa})$, and hip width (ACa). Descriptive statistical analysis were conducted for each variable. Analysis by sex and by locality were performed using Duncan's multiple comparison tests. Linear correlation analysis with sex was also tested. Difference in PT between male and female specimens was statistically significant. Comparisons by location revealed statistically significant differences $(p<0.05))$ for LC, AC, PT, LT, ACa, and LgCu. Duncan's multiple comparison tests show similarities in LC, PT and LT among specimens According to Duncan's multiple comparison tests, Creole cattle from Ngäble-Buglé Comarca is similar to the one found in El Valle de Anton - but different to Gualaca's - when LC, PT and LT are compared. Meanwhile, AC and $\mathrm{LgCu}$ values were found to be similar among Creole cattle from Ngäble-Buglé Comarca and from Gualaca; specimens from El Valle reported differences these measures. Cattle populations studied have no sexual dimorphism and show a degree of morphological homogeneity, which may be due to the Panamanian Creole cattle population being highly inbred.
\end{abstract}

Keywords: Zoometry; Creole cattle; Bovine; Panamanian cattle; Sex analysis.

\footnotetext{
${ }^{1}$ Trabajo realizado en el proyecto Conservación y Uso de la Biodiversidad Genética del Ganado Criollo Guaymi y Guabalá
} de Panamá. 


\section{Resumen}

El objetivo del estudio fue describir algunas variables zoométricas en el ganado bovino criollo de nuestro país. Durante el año 2011 se evaluaron 63 especímenes adultos, originarios de tres localidades diferentes: Gualaca (Chiriquî), El Valle de Antón (Coclé) y la Comarca Ngäbe Buglé (Chiriquî). Se midieron nueve variables zoométricas: largo de cara (LC), ancho de cara (AC), perímetro torácico (PT), largo total (LT), altura de la cruz (Acr), altura de grupa (AG), longitud del cuerpo (LgCu), longitud de la cadera ( $\mathrm{LgCa}$ ), ancho de la cadera (ACa). Se aplicó análisis estadístico descriptivo para cada una de las variables, una prueba comparativa múltiple de Duncan por sexo y por localidad, y por último, se realizó una correlación lineal por sexo. En la prueba comparativa de Duncan se observó diferencia significativa para PT en el análisis por sexo. Mientras que por localidad, se observaron diferencias significativas $(\mathrm{p}<0.05)$ para LC, AC, PT, LT, ACa y LgCu. Según la prueba de Duncan, LC, PT y LT asemeja al ganado bovino criollo de la Comarca Ngabe Buglé con El Valle de Antón y separa al de Gualaca; y AC y LgCu asocia al criollo de la Comarca Ngäbe Buglé con Gualaca y separa a El Valle. Se concluye que las poblaciones estudiadas no presentan marcado dimorfismo sexual y evidencian un grado de homogeneidad morfológica, lo cual puede deberse a que la población de bovino criollo panameño es altamente consanguínea.

Palabras clave: Zoometría; Ganado criollo; Bovino; Análisis por sexo, Ganadería panameña.

\section{Introducción}

Se denominan bovinos criollos a los descendientes puros y directos de los animales introducidos en los primeros años de la colonización americana. En Panamá la entrada se realizó a través de Santa María la Antigua del Darién en el Año 1521 (Jaén S., 1981). Actualmente el bovino criollo panameño se encuentra muy desigualmente distribuido en el territorio panameño siendo algunas comunidades de la comarca Ngöbe Buglé, área indígena y en el distrito de Gualaca; y en Guabalá, en la provincia de Chiriquí. Según Vargas (2005) el ganado criollo panameño presenta características que no se encuentran juntas en ningunas de las razas introducidas actualmente en el país, como lo son alta fertilidad, resistencia a parásitos y enfermedades, alta capacidad de pastoreo, aprovechamiento de pastoreo de baja calidad, mayor longevidad y otras.

Existen características fenotípicas poco influenciadas por el ambiente y que pueden aportar importantes evidencias de la diversidad animal como por ejemplo la conformación y el tamaño de la cabeza y de los cuernos (Alderson, 1992). La zoometría permite establecer patrones raciales a partir de la obtención de diferentes medidas corporales y analizar sus relaciones. Es una herramienta útil que contribuye a la caracterización y diferenciación racial (Pere-Miquel, 2006). Para ello es necesario realizar el estudio de la conformación del animal o morfología, que aporta una idea sobre la aptitud del mismo, abarcando una serie de medidas e índices corporales, siendo una herramienta valiosa para la clasificación de los animales en un grupo determinado (Alía, 1996). 
Invest. pens. crit.

Vol. 2, No. 5, septiembre-diciembre 2014.

pp. 26-33

\section{Materiales y métodos}

La recolección de los datos se realizó en tres localidades distintas, dos regiones de la provincia de Chiriquí y una región de la provincia de Coclé: Gualaca, geográficamente ubicada $8^{\circ} 31^{\prime} 48^{\prime \prime} \mathrm{N}, 82^{\circ}$ 17' 24" W y a una altura de 403 msnm; Comarca Ngäbe Buglé, geográficamente ubicada a $8^{\circ} 46^{\prime} 11^{\prime \prime}$ $\mathrm{N}, 81^{\circ} 44^{\prime} 2^{\prime \prime} \mathrm{W}$, y a una altura de 1,200 msnm. El Valle de Antón geográficamente ubicada a $8^{\circ} 36^{\prime}$ $2.88^{\prime \prime} \mathrm{N}, 80^{\circ} 7^{\prime} 49.08^{\prime \prime} \mathrm{W}$, con una elevación de 600 msnm.

Se registraron datos de nueve variables zoométricas en 63 bovinos criollos mayores de tres años de edad, 37 pertenecientes a la región de Gualaca, 12 de la región del Valle de Antón y 14 de la Comarca Ngäbe Buglé. Las variables zoométricas medidas en centímetros fueron: Largo de cabeza (LC) tomada desde la parte media del testuz hasta el comienzo de la mucosa del morro; ancho de cabeza (AC) medida de la distancia entre los ángulos mediales de los ojos; perímetro torácico (PT) medida del perímetro alrededor del pecho, pasando por la cruz y el esternón; largo total (LT) medido desde el encuentro hasta la punta del isquion; alzada a la cruz (Acr) medida de la distancia del suelo al punto más elevado de la cruz; alzada a la grupa (Ag) medida de la distancia del suelo al punto más elevado de la grupa; longitud del cuerpo $(\mathrm{LgCu})$, distancia entre la base de la inserción de la base de los cuernos y la inserción del maslo de la cola y el ancho de la cadera que es la distancia existente entre los dos puntos más sobresalientes de la masa muscular del anca. Para estimar el peso de los animales, se utilizó la formula descrita por Correa A (2005), indicada a continuación: Peso vivo $(\mathrm{PV})=(\mathrm{PT})(\mathrm{PT})(\mathrm{LgCu}) /$ 300. Esta fórmula utiliza las variables en pulgadas y expresa PV en libras (lb).

Sobre las medidas zoométricas del ganado criollo se aplicó un análisis estadístico descriptivo para observar la tendencia central de los datos. Posterior a esto, se realizó un análisis multivariado exploratorio de los datos colectados mediante una prueba comparativa múltiple de Duncan a fin de observar diferencias significativas entre el sexo y la localidad del ganado bovino criollo. Por último, se realizó una correlación lineal por sexo entre las variables analizadas para determinar el grado de dependencia entre ellas. El procesamiento de los datos se realizó a través del software Microsoft Excel.

\section{Resultados y discusión}

Los estadísticos descriptivos sobre la base de 63 bovinos criollos colectados en las regiones de Gualaca, Comarca Ngäbe-Buglé y El Valle de Antón, se muestran en la Tabla 1. Todas las estadísticas están dadas en cm, excepto el coeficiente de variabilidad $(\mathrm{Cv})$ que está dado en porcentaje (\%).

El largo promedio de la cara (LC) para estos bovinos criollos es de $43.14 \mathrm{~cm}$, valor que con una confiabilidad de $95 \%$ varía desde un mínimo de 42.02 hasta $44.22 \mathrm{~cm}$. Los resultados de la investigación muestran una desviación estándar de $4.377 \mathrm{~cm}$; el valor del coeficiente de variabilidad de 10.14\% muestra que los errores fueron bien controlados en esta muestra. El ancho promedio de la cara (AC) es de $21.05 \mathrm{~cm}$, que con una confiabilidad de 95\% varía desde un mínimo de 20.33 hasta $21.77 \mathrm{~cm}$. 
Los resultados de la investigación muestran una desviación estándar de $2.915 \mathrm{~cm}$; el valor del coeficiente de variabilidad de $13.85 \%$ muestra que los errores fueron bien controlados en esta muestra.

El perímetro promedio del tórax (PT) es de $149.5 \mathrm{~cm}$, valor que con una confiabilidad de 95\% varía desde un mínimo de 144.43 hasta $154.57 \mathrm{~cm}$. Los resultados de la investigación muestran una desviación estándar de $20.536 \mathrm{~cm}$; el valor del coeficiente de variabilidad de $13.73 \%$ muestra que los errores fueron bien controlados en esta muestra. La longitud total promedio (LT) es de $161.46 \mathrm{~cm}$, valor que con una confiabilidad de 95\% varía desde un mínimo de 154.67 hasta $168.25 \mathrm{~cm}$. Los resultados de la investigación muestran una desviación estándar de $27.501 \mathrm{~cm}$; el valor del coeficiente de variabilidad de $17.03 \%$ muestra que los errores fueron bien controlados en esta muestra.

La altura promedio de la cruz (Acr) es de $116.855 \mathrm{~cm}$, valor que con una confiabilidad de 95\% varía desde un mínimo de 113.97 hasta 119,74 cm. Los resultados de la investigación muestran una desviación estándar de $11.685 \mathrm{~cm}$; el valor del coeficiente de variabilidad de $9.99 \%$ muestra que los errores fueron bien controlados en esta muestra. El ancho promedio de la grupa (AG) es de $117.729 \mathrm{~cm}$, valor que con una confiabilidad de 95\% varía desde un mínimo de 112.07 hasta $123.39 \mathrm{~cm}$. Los resultados de la investigación muestran una desviación estándar de $22.914 \mathrm{~cm}$; el valor del coeficiente de variabilidad de $19.46 \%$ muestra que los errores fueron bien controlados en esta muestra.

La longitud promedio del cuerpo $(\mathrm{LgCu})$ es de $125.351 \mathrm{~cm}$, valor que con una confrabilidad de 95\% varía desde un mínimo de 120.474 hasta $130.227 \mathrm{~cm}$. Los resultados de la investigación muestran una desviación estándar de $18.378 \mathrm{~cm}$; el valor del coeficiente de variabilidad de $14.66 \%$ muestra que los errores fueron bien controlados en esta muestra.

La longitud promedio de la cadera $(\mathrm{LgCa})$ es de $36.395 \mathrm{~cm}$, valor que con una confiabilidad de 95\% varía desde un mínimo de 34.942 hasta $37.849 \mathrm{~cm}$. Los resultados de la investigación muestran una desviación estándar de $5.005 \mathrm{~cm}$; el valor del coeficiente de variabilidad de $13.75 \%$ muestra que los errores fueron bien controlados en esta muestra. El ancho promedio de la cadera (ACa) es de 36.417 $\mathrm{cm}$, valor que con una confiabilidad de 95\% varía desde un mínimo de 34.237 hasta $38.596 \mathrm{~cm}$. Los resultados de la investigación muestran una desviación estándar de $7.505 \mathrm{~cm}$; el valor del coeficiente de variabilidad de $20.61 \%$ muestra que los errores fueron bien controlados en esta muestra.

Tabla 1. Análisis estadístico descriptivo de las medidas zoométricas.

\begin{tabular}{ccccccc}
\hline Variable & Media & $\begin{array}{c}\text { Desviación } \\
\text { Estándar }\end{array}$ & $\begin{array}{c}\text { Límite } \\
\mathbf{M i ́ n i m o} \\
\mathbf{9 5 \%}\end{array}$ & $\begin{array}{c}\text { Límite } \\
\text { Máximo } \\
\mathbf{9 5 \%}\end{array}$ & $\begin{array}{c}\text { Coeficiente } \\
\text { de variación } \\
\mathbf{\%}\end{array}$ & $\begin{array}{c}\text { Intervalo de } \\
\text { Confianza } \\
\mathbf{9 5 \%}\end{array}$ \\
\hline $\mathbf{L C}$ & 43.14 & 4.377 & 42.06 & 44.22 & 10.14 & $+/-1.080$ \\
$\mathbf{A C}$ & 21.047 & 2.915 & 20.33 & 21.77 & 13.85 & $+/-0.719$ \\
$\mathbf{P T}$ & 149.5 & 20.536 & 144.43 & 154.57 & 13.73 & $+/-5.071$ \\
$\mathbf{L T}$ & 161.46 & 27.501 & 154.67 & 168.25 & 17.03 & $+/-6.791$ \\
$\mathbf{A C R}$ & 116.855 & 11.685 & 113.97 & 119,74 & 9.99 & $+/-2.885$ \\
$\mathbf{A G}$ & 117.729 & 22.914 & 112.07 & 123.39 & 19.46 & $+/-5.658$ \\
$\mathbf{L g C u}$ & 125.351 & 18.378 & 120.474 & 130.227 & 14.66 & $+/-4.877$ \\
$\mathbf{L g C a}$ & 36.395 & 5.005 & 34.942 & 37.849 & 13.75 & $+/-1.454$ \\
$\mathbf{A C a}$ & 36.417 & 7.505 & 34.237 & 38.596 & 20.61 & $+/-2.179$ \\
\hline
\end{tabular}


Invest. pens. crit.

Vol. 2, No. 5, septiembre-diciembre 2014.

pp. 26-33

El análisis exploratorio de los datos mediante la prueba de Duncan por el efecto del sexo, demostró que solo existen diferencias significativas $(\mathrm{p}<0.05)$ para la variable perímetro torácico $(\mathrm{PT})$ como se muestra en la Tabla 2, resultando el PT como el mejor indicador del peso vivo (PV), igual como lo han señalado otros investigadores (Mancheha et al., 2002). Las demás variables presentaron diferencias no significativas ( $\mathrm{p}>0.05)$, por lo que PT se analizó por separado para la prueba múltiple de Duncan por localidad. La relación entre PT y PV, se explica claramente en la Gráfica 1, donde se marca notoriamente una relación directa con un $\mathrm{R}^{2}=0.9189$.

\begin{tabular}{cccccc} 
Tabla 2. Resumen de análisis de varianza de las medidas zoométricas según el sexo. \\
\cline { 2 - 6 } & $\mathbf{C v}$ & $\mathbf{C M}$ & $\mathbf{F}$ & $\mathbf{P}$ & Sig. \\
\hline $\mathbf{L C}$ & 10.03 & 46.3195 & 2.48 & 0.1208 & \\
$\mathbf{A C}$ & 13.56 & 29.7782 & 3.65 & 0.0606 & \\
$\mathbf{P T}(\mathbf{c m})$ & 13.32 & 1955.6630 & 4.93 & 0.0301 & $*$ \\
$\mathbf{L T}$ & 16.90 & 1379.5926 & 1.85 & 0.1792 & \\
$\mathbf{A c r}$ & 9.94 & 240.8602 & 1.79 & 0.1864 & \\
$\mathbf{A G}$ & 13.78 & 170.6327 & 0.65 & 0.4249 & \\
$\mathbf{L g C u}$ & 13.68 & 37.6094 & 1.52 & 0.2242 & \\
$\mathbf{L g C a}$ & 20.36 & 118.9597 & 2.16 & 0.1481 & \\
$\mathbf{A C a}$ & 18.67 & 711.8424 & 1.34 & 0.2527 & \\
\hline
\end{tabular}

Figura 1.Relación entre las variables PTy PV.

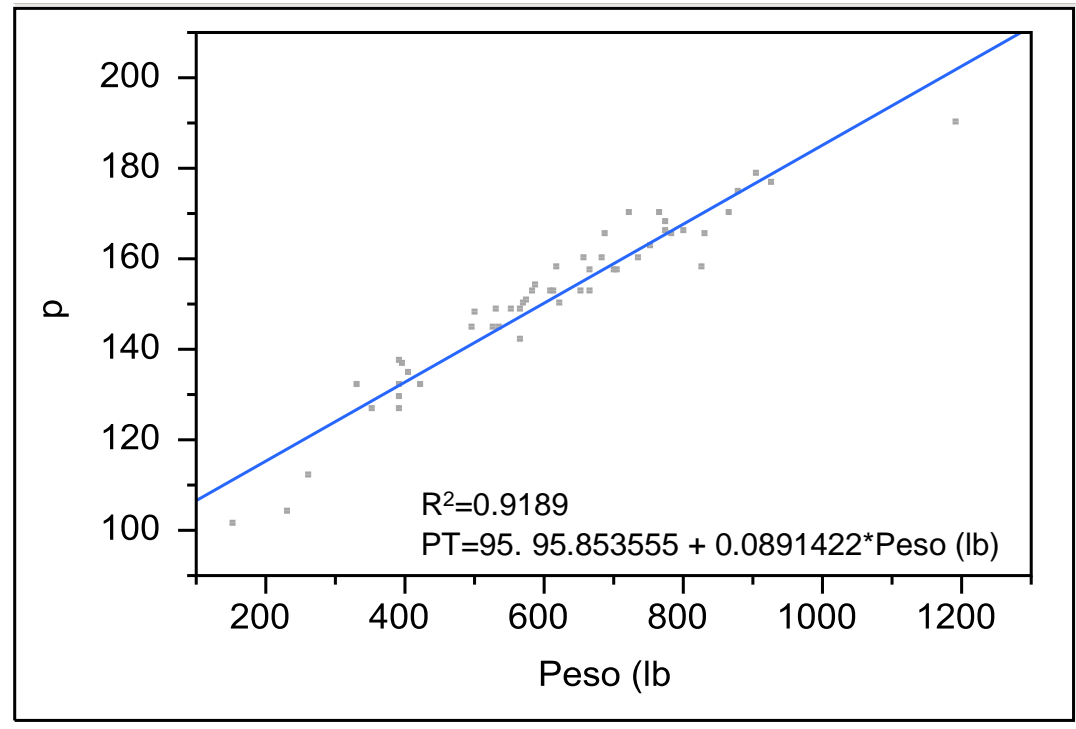

Para la prueba múltiple de Duncan por localidad, se evaluó el ganado bovino criollo originario de tres localidades diferentes, Gualaca, Chiriquí; El Valle de Antón, Coclé y La Comarca Ngäbe Buglé, Chiriquí. De esta prueba, se observaron diferencias significativas $(\mathrm{p}<0.05)$ para las variables LC, AC, PT, LT, ACa y LgCu, y diferencias no significativas ( $p>0.05$ ) para las variables Acr, LgCa y Ag. Probablemente, las diferencias significativas existentes entre las variables está influenciada por factores inherentes al individuo, como el sexo, la edad y el ambiente en el cual se desenvuelven. 
Tabla 3. Resumen de análisis de varianza de las medidas zoométricas según la localidad.

\begin{tabular}{cccccc}
\hline & $\mathbf{C v}$ & $\mathbf{C M}$ & $\mathbf{F}$ & $\mathbf{P}$ & Sig. \\
\hline $\mathbf{L C}$ & 9.54 & 85.7067 & 5.06 & 0.0093 & $*$ \\
$\mathbf{A C}$ & 12.31 & 62.1363 & 9.26 & 0.0003 & $*$ \\
$* * \mathbf{P T}(\mathbf{c m})$ & 10.09 & 2376.4595 & 10.19 & 0.0002 & $*$ \\
$\mathbf{L T}$ & 13.64 & 7666.0724 & 15.80 & 0.0001 & $*$ \\
$\mathbf{A c r}$ & 9.84 & 262.3964 & 1.98 & 0.1466 & \\
$\mathbf{A G}$ & 13.32 & 966.1736 & 3.93 & 0.0535 & \\
$\mathbf{L g C u}$ & 17.56 & 2290.1374 & 4.86 & 0.0114 & $*$ \\
$\mathbf{L g C a}$ & 13.56 & 57.5069 & 2.36 & 0.1312 & \\
$\mathbf{A C a}$ & 16.42 & 1002.7778 & 28.04 & 0.0001 & $*$ \\
\hline
\end{tabular}

El resumen de agrupamiento de las medias de las medidas zoométricas por Duncan (Tabla 4), asocia en las variables LC, PT y LT al ganado criollo de la Comarca Ngäbe Buglé con el ganado criollo de El Valle y separa al ganado criollo de Gualaca. Sin embargo, asocia al criollo de la Comarca Ngäbe Buglé con los de Gualaca y separa al criollo de El Valle en las variables AC y $\mathrm{LgCu}$.

Las variables asociadas del ganado criollo de la Comarca y Gualaca, probablemente se deba a que en el año 1990 fueron trasladados cinco vientres de ganado criollo de las montañas de San Félix, perteneciente a la Comarca Ngäbe Buglé a la localidad de Gualaca con la finalidad de extraer y congelar semen, conformado un banco de recurso genético para iniciar los procesos de conservación de la raza criolla panameña (González M et al, 2009b).

\begin{tabular}{cccc} 
Tabla 4. Agrupamiento de medias de las medidas zoométricas por localidad. \\
\cline { 2 - 4 } & \multicolumn{4}{c}{ Medias } \\
\cline { 2 - 4 } LC & $\underline{\text { El Valle }}$ & $\frac{\text { Comarca NB }}{45.917 \mathrm{a}}$ & Gualaca \\
AC & $23.917 \mathrm{a}$ & $20.714 \mathrm{a}$ & $41.838 \mathrm{~b}$ \\
**PT (cm) & $167.091 \mathrm{a}$ & $156.195 \mathrm{a}$ & $14.243 \mathrm{~b}$ \\
$\mathbf{L T}$ & $190.500 \mathrm{a}$ & $173.400 \mathrm{a}$ & $150.430 \mathrm{~b}$ \\
$\mathbf{A c r}$ & $120.571 \mathrm{a}$ & $119.917 \mathrm{a}$ & $114.389 \mathrm{a}$ \\
$\mathbf{A G}$ & $125.500 \mathrm{a}$ & - & $115.139 \mathrm{a}$ \\
$\mathbf{L g C u}$ & $140.583 \mathrm{a}$ & $123.222 \mathrm{~b}$ & $118.028 \mathrm{~b}$ \\
$\mathbf{L g C a}$ & $34.500 \mathrm{a}$ & - & $37.028 \mathrm{a}$ \\
$\mathbf{A C a}$ & $44.333 \mathrm{a}$ & - & $33.778 \mathrm{~b}$ \\
\hline
\end{tabular}

Del análisis de correlación lineal efectuado a las medidas zoométricas según el sexo, se observó que para las hembras existe una alta correlación $(\mathrm{r} \geq 0.7)$ entre la mayoría de las variables, siendo más representativa la relación entre (LT) y (PT), (LT) y (AC), y (PT) y (ACa), según la Tabla 5. Lo que evidencia un grado de homogeneidad morfológica y que en términos medios, existen rasgos distintivos que las caracterizan. 
Invest. pens. crit.

Vol. 2, No. 5, septiembre-diciembre 2014.

pp. $26-33$

Tabla 5. Análisis de correlación entre las medidas zoométricas de las hembras.

\begin{tabular}{cccccccccc}
\hline & LC & AC & PT & LT & Acr & AG & LgCu & LgCa & ACa \\
\hline LC & 1.0000 & & & & & & & & \\
AC & 0.5472 & 1.0000 & & & & & & & \\
PT & 0.7559 & 0.7193 & 1.0000 & & & & & & \\
LT & 0.7307 & 0.7361 & $0.9198^{*}$ & 1.0000 & & & & & \\
Acr & 0.5927 & 0.4773 & 0.6976 & 0.6426 & 1.0000 & & & & \\
AG & 0.4674 & 0.3119 & 0.4421 & 0.3977 & 0.2516 & 1.0000 & & & \\
$\mathbf{L g C u}$ & 0.5315 & 0.5764 & 0.7249 & 0.6961 & 0.6119 & 0.3003 & 1.0000 & & \\
$\mathbf{L g C a}$ & 0.3987 & 0.1852 & 0.3823 & 0.3579 & 0.7256 & 0.1483 & 0.3679 & 1.0000 & \\
ACa & 0.7394 & 0.6894 & $0.9206^{*}$ & $0.9147 *$ & 0.6848 & 0.3514 & 0.7219 & 0.3281 & 1.0000 \\
\hline
\end{tabular}

Igualmente para los machos, existe una alta correlación $(r \geq 0.7)$ entre casi todas las variables, como se observa en la Tabla 6. Lo que nos indican que las medidas zoométricas estudiadas no muestran, diferencias, entre ambos sexos de la raza criolla, no estableciendo un marcado dimorfismo sexual contario a lo reportado por (Martínez et al 2007).

Tabla 6. Análisis de correlación entre las medidas zoométricas de los machos.

\begin{tabular}{cccccccccc}
\hline & LC & AC & PT & LT & Acr & AG & LgCu & LgCa & ACa \\
\hline LC & 1.0000 & & & & & & & & \\
AC & 0.7020 & 1.0000 & & & & & & & \\
PT & 0.9651 & 0.7388 & 1.0000 & & & & & & \\
LT & 0.9094 & 0.7189 & 0.9635 & 1.0000 & & & & & \\
Acr & 0.9551 & 0.7597 & 0.8988 & 0.8682 & 1.0000 & & & & \\
AG & 0.9296 & 0.8587 & 0.9148 & 0.9179 & 0.9673 & 1.0000 & & & \\
LgCu & 0.8925 & 0.6867 & 0.9532 & 0.9913 & 0.7982 & 0.8713 & 1.0000 & & \\
LgCa & 0.9573 & 0.5010 & 0.8816 & 0.7941 & 0.9221 & 0.8302 & 0.7319 & 1.0000 & \\
ACa & 0.9395 & 0.7067 & 0.9578 & 0.9974 & 0.8926 & 0.9334 & 0.9799 & 0.8078 & 1.0000 \\
\hline
\end{tabular}

\section{Conclusión}

Se concluye que las poblaciones estudiadas no presentan marcado dimorfismo sexual y evidencian un grado de homogeneidad morfológica, esto se debe a que la población de bovino criollo panameño es altamente consanguínea, lo cual coincide con recomendaciones dadas.

\section{Referencias}

Alderson, L. (1992). The categorization of types and breeds of cattle in Europe. Archivos de Zootecnia, 41, 325-334.

Alía R, M J. (1996). La base animal en el ganado caprino. Producción ovina y caprina.

Buxadé, C. (Coord.) (1996). Zootecnia. Bases de producción animal (Tomo VIII). Madrid, España: Ediciones Mundi-Prensa. 
González M, RA; Guerra M, P; Ríos, R y De León G, R. (2009a). Comportamiento productivo y reproductivo del ganado bovino criollo de Panamá bajo condiciones mejoradas en la estación experimental de Gualaca. Panamá: Instituto de Investigación Agropecuaria de Panamá. Folleto Técnico.

González M, RA; Guerra M, P; Ríos, R; y De León G, R. (2009b). Origen y Características raciales del ganado bovino criollo panameño. Panamá: Instituto de Investigación Agropecuaria de Panamá. Folleto Técnico.

Jaén S, O. (1981). Nuevos hombres y ganado y su impacto en el paisaje geográfico panameño entre 1500 y 1980, en Hombres y Ecología en Panamá. Panamá: Editorial Universitaria - STRI.

Manchencha, L; Angulo, J; y Manrique, LP. (2002). Estudio bovinométrico y relaciones entre medidas corporales y el peso vivo en la raza Lucerna. Revista Colombiana Ciencia Pecuaria, 15(1), 80- 87.

Martínez M, R; Fernández T, E; Abbiati C, N; y Broccoli R, A. (2007). Caracterización zoométrica de bovinos criollos: patagónicos vs noroeste argentino. Rev. MVZ Córdoba, 12(2), 1042-1049.

Parés i Casanova, PM. (2006). Medidas e índices cefálicos en la raza bovina "Bruna Dels Pirineus". Revista electrónica veterinaria. REDVET.

http://www.veterinaria.org/revistas/redvet/n090906.html 